\title{
Numerical Methods for a Class of Differential Algebraic Equations
}

\author{
Lei Ren and Yuan-Ming Wang \\ Department of Mathematics, Shanghai Key Laboratory of Pure Mathematics and Mathematical Practice, \\ East China Normal University, Shanghai 200241, China
}

Correspondence should be addressed to Lei Ren; renleipost@163.com

Received 28 November 2016; Accepted 11 April 2017; Published 8 June 2017

Academic Editor: Fazal M. Mahomed

Copyright (C) 2017 Lei Ren and Yuan-Ming Wang. This is an open access article distributed under the Creative Commons Attribution License, which permits unrestricted use, distribution, and reproduction in any medium, provided the original work is properly cited.

\begin{abstract}
This paper is devoted to the study of some efficient numerical methods for the differential algebraic equations (DAEs). At first, we propose a finite algorithm to compute the Drazin inverse of the time varying DAEs. Numerical experiments are presented by Drazin inverse and Radau IIA method, which illustrate that the precision of the Drazin inverse method is higher than the Radau IIA method. Then, Drazin inverse, Radau IIA, and Padé approximation are applied to the constant coefficient DAEs, respectively. Numerical results demonstrate that the Padé approximation is powerful for solving constant coefficient DAEs.
\end{abstract}

\section{Introduction}

The general differential algebraic equations (DAEs)

$$
F\left(t, x(t), x^{\prime}(t)\right)=0
$$

arise in many applications, circuit analysis, control theory, chemical process simulations, singular perturbation, constrained mechanical systems of rigid bodies, and so forth [13]. In [4-6], the numerical solutions of constant coefficient DAEs were studied by Golub et al. The linear time varying of DAE

$$
A(t) x^{\prime}(t)+B(t) x(t)=f(t)
$$

defined on the interval $I$ is important in understanding general DAEs. It exhibits most of the behavior found in the nonlinear case that is not already present in the constant coefficient case, yet the linearity facilitates the analysis. In spite of this, many aspects of the theory and numerical solution of (2) have only been resolved within the last few years. Some questions remain open.

In [7], one canonical form for higher index linear time varying singular systems has been presented. In [8-10], with the DAEs being tractable with indexes 2 and 3 some results were proposed. It was shown that either 2 or index 3 tractability causes the homogeneous equations with respect to (2) to provide a finite dimensional space of solutions. In [11], Song had treated general higher index time varying linear DAEs in terms of matrix pencils and investigated their basic properties and their solvability.

Above these results, the numerical solutions of DAEs seldom are involved. In this paper, we shall consider the homogeneous equations with respect to DAEs:

$$
A(t) x^{\prime}(t)+B(t) x(t)=0 .
$$

In this paper, we shall consider the constant coefficient and time varying DAEs with different numerical methods. In Section 2, we present some definitions which will be used in the proofs of our main theorems. In Section 3, a finite algorithm for the computation of the Drazin inverse of the time varying singular matrix $A(t)$ is presented. Drazin inverse is applied to (3) corresponding to the difference equations; we will establish sufficient and necessary conditions for the solution of (3). In Section 4, the numerical treatment with the corresponding difference format is presented; then the numerical solutions of constant coefficient DAEs are presented by Padé approximation and the implicit RungeKutta method. In Section 5, some numerical examples and error estimates are proposed. 


\section{Preliminaries}

We first introduce some definitions of the Drazin inverse of a matrix.

Definition 1. Let $A \in \mathbb{C}^{n \times n}$. If the smallest positive integer $k$ such that

$$
\operatorname{rank}\left(A^{k+1}\right)=\operatorname{rank}\left(A^{k}\right)
$$

holds it is called the index of $A$ and is denoted by $k=\operatorname{Ind}(A)$. If $\mathrm{A}$ is nonsingular, then $\operatorname{Ind}(A)=0$; else if $A$ is singular, then $\operatorname{Ind}(A) \geq 1$.

Definition 2. Let $A \in \mathbb{C}^{n \times n}$ and $\operatorname{Ind}(A)=k$. Then the matrix $X \in \mathbb{C}^{n \times n}$ satisfying

$\left(1^{k}\right)$

$$
A^{k} X A=A^{k}
$$

(2)

$$
X A X=X
$$

(3)

$$
A X=X A
$$

is called the Drazin inverse of $A$ and is denoted by $X=A_{d}$.

Consider the following singular differential and corresponding difference equations

$$
\begin{aligned}
A(t) x^{\prime}+B(t) x(t) & =0, \\
A(t) x_{n+1} & =B(t) x_{n} .
\end{aligned}
$$

Suppose $t_{n+1}=t_{n}+h ; h$ is sufficient small. Then

$$
A(t) \frac{x\left(t_{n+1}\right)-x\left(t_{n}\right)}{t_{n+1}-t_{n}} \approx A(t) x\left(t_{n}\right)=-B(t) x\left(t_{n}\right) .
$$

Therefore, the approximation of $A x^{\prime}$ is given by

$$
A(t) x_{n+1}=A(t) x_{n}-h B(t) x_{n} .
$$

Let $C(t)=(A(t)-h B(t))$; we can get the difference equations $A(t) x_{n+1}=C(t) x_{n}$ like (8). When $A(t)$ is a singular matrix, things can happen that are impossible when $A(t)^{-1}$ exists.

Definition 3. For $A(t), B(t) \in \mathbb{C}^{n \times n}(t)$ and $t_{0} \in R$, the vector $c \in \mathbb{C}^{n}$ is called a consistent initial vector associated with $t_{0}$ for the equation $A(t) x^{\prime}(t)+B(t) x(t)=f(t)$ when the initial problem $A(t) x^{\prime}(t)+B(t) x(t)=f(t), x\left(t_{0}\right)=c, n=1,2, \ldots$, processes at least one solution.

Definition 4. The equation $A(t) x^{\prime}(t)+B(t) x(t)=f(t)$ is said to tractable at the point $t_{0}$ if the initial value problem $A(t) x^{\prime}(t)+B(t) x(t)=f(t), x\left(t_{0}\right)=c$ has a unique solution for each consistent initial vector $c$ associated with $t_{0}$.
Definition 5. For $A(t), B(t) \in \mathbb{C}^{n \times n}(t)$ and $f_{n} \in \mathbb{C}^{n}$, the vector $c \in \mathbb{C}^{n}$ is called a consistent initial vector for the difference equation $A(t) x_{n+1}=B(t) x_{n}+f_{n}$ if the initial value problem $A(t) x_{n+1}=B(t) x_{n}+f_{n}, x_{0}=c, n=1,2, \ldots$, has a solution for $x_{n}$.

Definition 6. The difference equation $A(t) x_{n+1}=B(t) x_{n}+f_{n}$ is said to be tractable if the initial value problem $A(t) x_{n+1}=$ $B(t) x_{n}+f_{n}, x_{0}=c, n=1,2, \ldots$, has a unique solution for each consistent initial vector $c$.

\section{Time Varying Differential Algebraic Equations}

Lemma 7 (see [12]). For $A(t) \in R(t)^{n \times n}, \operatorname{Ind}(A(t))=k$, and

$$
\begin{aligned}
g(\lambda, t)= & \operatorname{det}\left(\lambda I-A^{k+1}(t)\right) \\
= & g_{0}(t) \lambda^{n}+g_{1}(t) \lambda^{n-1}+\cdots+g_{n-1}(t) \lambda \\
& +g_{n}(t)
\end{aligned}
$$

is characteristic polynomial of $A^{k+1}(t)$, where $g_{0}(t)=1$. Then $g_{r+1}(t)=\cdots=g_{n}(t)=0, g_{r}(t) \neq 0$, and

$$
\begin{aligned}
& A_{d}(t)=-g_{r}^{-1}(t) A^{k}(t)\left[\left(A^{k+1}(t)\right)^{r-1}\right. \\
& \quad+g_{1}(t)\left(A^{k+1}(t)\right)^{r-2}+\cdots+g_{r-2}(t)\left(A^{k+1}(t)\right) \\
& \left.\quad+g_{r-1}(t) I_{n}\right],
\end{aligned}
$$

where $r=\operatorname{rank}(A(t))$.

Next, the algorithm for the computation of the Drazin inverse of a polynomial matrix is presented as follows.

Step 1. $F_{i}(t)$ and $g_{i}(t)$ are determined by the recursive relationship as follows:

$$
\begin{aligned}
& F_{i+1}(t)=A^{k+1}(t) F_{i}(t)+g_{i}(\mathrm{t}) I, \\
& g_{i+1}(t)=-\frac{\operatorname{tr}\left(A^{k+1}(t) F_{i+1}(t)\right)}{(i+1)},
\end{aligned}
$$

and initial conditions are

$$
\begin{aligned}
& F_{1}(t)=I_{n}, \\
& g_{1}(t)=-\operatorname{tr}\left(A^{k+1}(t)\right) .
\end{aligned}
$$

Step 2. From $\operatorname{rank}\left(A^{k}(t)\right)=\operatorname{rank}\left(A^{k+1}(t)\right)=r$, we can see that $g_{r+1}(t)=\cdots=g_{n}(t)=0, g_{r}(t) \neq 0$. Then

$$
\begin{aligned}
& A_{d}(t)=-g_{r}^{-1}(t) A^{k}(t)\left[\left(A^{k+1}(t)\right)^{r-1}\right. \\
& \quad+g_{1}(t)\left(A^{k+1}(t)\right)^{r-2}+\cdots+g_{r-2}(t)\left(A^{k+1}(t)\right) \\
& \left.\quad+g_{r-1}(t) I_{n}\right]=-g_{r}^{-1}(t) A^{k}(t) F_{r}(t) .
\end{aligned}
$$

The algorithm could be performed by the symbol computation package of Matlab. 
We are now ready to give solutions on corresponding singular difference equation. First of all, in order to establish a sufficient and necessary condition, the following lemma is important.

Lemma 8. Let $A(t), B(t) \in \mathbb{C}^{n \times n}(t)$. Assume that there exists $\lambda \in \mathbb{C}$, such that $(\lambda A(t)+B(t))^{-1}$ exists, and let

$$
\begin{aligned}
\widehat{A}_{\lambda}(t) & =(\lambda A(t)+B(t))^{-1} A(t), \\
\widehat{B}_{\lambda}(t) & =(\lambda A(t)+B(t))^{-1} B(t) .
\end{aligned}
$$

Then $\widehat{A}_{\lambda}(t) \widehat{B}_{\lambda}(t)=\widehat{B}_{\lambda}(t) \widehat{A}_{\lambda}(t)$.

Proof. If there exists $\lambda \in \mathbb{C}$ such that $(\lambda A(t)+B(t))^{-1}$ exists, then

$$
\begin{aligned}
\lambda \widehat{A}_{\lambda}(t)+\widehat{B}_{\lambda}(t)= & \lambda(\lambda A(t)+B(t))^{-1} A(t) \\
& +(\lambda A(t)+B(t))^{-1} B(t) \\
= & (\lambda A(t)+B(t))^{-1}(\lambda A(t)+B(t)) \\
= & I .
\end{aligned}
$$

Thus

$\widehat{B}_{\lambda}(t) \widehat{A}_{\lambda}(t)=\left(I-\lambda \widehat{A}_{\lambda}(t)\right) \widehat{A}_{\lambda}(t)=\widehat{A}_{\lambda}(t)-\lambda \widehat{A}_{\lambda}(t)^{2}=$ $\widehat{A}_{\lambda}(t)\left(I-\lambda \widehat{A}_{\lambda}(t)\right)=\widehat{A}_{\lambda}(t) \widehat{B}_{\lambda}(t)$. Consequently, $\widehat{A}_{\lambda}(t) \widehat{B}_{\lambda}(t)=$ $\widehat{B}_{\lambda}(t) \widehat{A}_{\lambda}(t)$.

Theorem 9. For $A(t), B(t) \in \mathbb{C}^{n \times n}(t)$, the homogeneous difference equation

$$
A(t) x_{n+1}=B(t) x_{n}
$$

is tractable if and only if there exists $\lambda \in \mathbb{C}$ such that $(\lambda A(t)+$ $B(t))^{-1}$ exists.

Proof. We first prove the sufficiency. Let $\widehat{A}_{\lambda}(t)$ and $\widehat{B}_{\lambda}(t)$ be defined as those in (27). Clearly $A(t) x_{n+1}=B(t) x_{n}$ is tractable if and only if $\widehat{A}_{\lambda}(t) x_{n+1}=\widehat{B}_{\lambda}(t) x_{n}$ is tractable.

Since $\lambda \widehat{A}_{\lambda}(t)+\widehat{B}_{\lambda}(t)=I, \exists$ invertible matrix $T(t) \epsilon$ $\mathbb{C}^{n \times n}(t)$, so that

$$
\begin{aligned}
T^{-1}(t) \widehat{A}_{\lambda}(t) T(t) & =\left(\begin{array}{cc}
C(t) & 0 \\
0 & N(t)
\end{array}\right), \\
T^{-1}(t) \widehat{B}_{\lambda}(t) T(t) & =\left(\begin{array}{cc}
I-\lambda C(t) & 0 \\
0 & I-\lambda N(t)
\end{array}\right) \\
& =\left(\begin{array}{cc}
\widehat{B}_{1}(t) & 0 \\
0 & \widehat{B}_{2}(t)
\end{array}\right),
\end{aligned}
$$

where $C(t)$ is invertible and $N(t)$ is nilpotent of index $k$. Let $x_{n}=T(t) y_{n}(t)$. Then the differential equation becomes

$$
\begin{aligned}
& \left(\begin{array}{cc}
C(t) & 0 \\
0 & N(t)
\end{array}\right)\left(\begin{array}{c}
y_{n+1}^{(1)}(t) \\
y_{n+1}^{(2)}(t)
\end{array}\right) \\
& \quad=\left(\begin{array}{cc}
I-\lambda C(t) & 0 \\
0 & I-\lambda N(t)
\end{array}\right)\left(\begin{array}{c}
y_{n}^{(1)}(t) \\
y_{n}^{(2)}(t)
\end{array}\right),
\end{aligned}
$$

or

$$
\begin{aligned}
& C(t) y_{n+1}^{(1)}(t)=(I-\lambda C(t)) y_{n}^{(1)}(t), \\
& N(t) y_{n+1}^{(2)}(t)=(I-\lambda N(t)) y_{n}^{(2)}(t) .
\end{aligned}
$$

Since $C(t)$ is invertible, $C(t) y_{n+1}^{(1)}(t)=(I-\lambda C(t)) y_{n}^{(1)}(t)$ is tractable. Thus it suffices to show that the second equation of is tractable. Let $k=\operatorname{Ind}(N(t))$ and multiply (32) by $N^{k-1}(t)$. Then $(I-\lambda N(t)) N^{k-1} y_{n}^{(2)}=0$, and hence $N^{k-1} y_{n}^{(2)}=0$. So $N^{k-1} y_{n+1}^{(2)}=0$. Multiply (32) again by $N^{k-2}$. Then $N^{k-1} y_{n+1}^{(2)}=$ $(I-\lambda N(t)) N^{k-2} y_{n}^{(2)}$, so $N^{k-2} y_{n}^{(2)}=0$. Continuing in this manner, we get $y_{n}^{(2)}(t)=0$ and $N y_{n+1}^{(2)}+(I-\lambda N(t)) y_{n}^{(2)}=0$ is trivially tractable.

For the necessity, assume that $A(t) x_{n+1}=B(t) x_{n}$ is tractable. We need to show that there is a $\lambda \in \mathbb{C}$ such that $\lambda A(t)+B(t)$ is invertible. Assume that this is not true. Then $\lambda A(t)+B(t)$ is singular for all $\lambda \in \mathbb{C}$. This means that, for each $\lambda \in \mathbb{C}$, there is a vector $v_{\lambda}(t) \in \mathbb{C}(t)$ such that $(\lambda A(t)+$ $B(t)) v_{\lambda}(t)=0$ and $v_{\lambda}(t) \neq 0$. Let $\left\{v_{\lambda_{1}}(t), v_{\lambda_{2}}(t), \ldots, v_{\lambda_{s}}(t)\right\}$ be a finite linearly dependent set of such vectors. Let $x_{n}^{\left(\lambda_{i}\right)}=$ $\lambda_{i}^{n} v_{\lambda_{i}}(t)$ and let $\left\{\alpha_{1}(t), \alpha_{2}(t), \ldots, \alpha_{s}(t)\right\} \subseteq \mathbb{C}(t)$ be such that $\sum_{i=1}^{s} \alpha_{i} v_{\lambda_{i}}(t)=0$, where not all $\alpha_{i}$ are 0 . Then $z_{n}=\sum_{i=1}^{s} \alpha_{i} x_{n}^{\left(\lambda_{i}\right)}$ is not identically zero and is easily seen to be a solution of (18). However, $z_{0}=\sum_{i=1}^{s} \lambda_{i} v_{\lambda_{i}}(t)=0$. Thus there are two different solutions, namely, $z_{n}$ and 0 , which satisfy the initial condition $x_{0}=0$. Therefore, it is not tractable at $n=0$, which contradicts our hypothesis. Hence, $(\lambda A(t)+B(t))^{-1}$ exists for some $\lambda \in \mathbb{C}$.

The next lemma will be used to show that the solution of the difference equation is independent of the scalar $\lambda$ which is used in the expressions $(\lambda A(t)+B(t))^{-1}$ and $(\lambda A(t)-B(t))^{-1}$.

Lemma 10. Assume that $A(t), B(t) \in \mathbb{C}^{n \times n}(t)$ are such that there exists $\lambda \in \mathbb{C}$ such that $(\lambda A(t) \pm B(t))^{-1}$ exists. Let $\widehat{A}_{\lambda}(t)=$ $(\lambda A(t) \pm B(t))^{-1} A(t), \widehat{B}_{\lambda}(t)=(\lambda A(t) \pm B(t))^{-1} B(t)$. For all $\alpha, \beta \in \mathbb{C}$ for which $(\alpha A(t) \pm B(t))^{-1}$ and $(\beta A(t) \pm B(t))^{-1}$ exist, the following statements are true:

$$
\begin{gathered}
\widehat{A}_{\alpha}^{d}(t) \widehat{A}_{\alpha}(t)=\widehat{A}_{\beta}^{d}(t) \widehat{A}_{\beta}(t), \\
\widehat{A}_{\alpha}^{d}(t) \widehat{B}_{\alpha}(t)=\widehat{A}_{\beta}^{d}(t) \widehat{B}_{\beta}(t), \\
\widehat{A}_{\alpha}(t) \widehat{B}_{\alpha}^{d}(t)=\widehat{A}_{\beta}(t) \widehat{A}_{\beta}^{d}(t), \\
\operatorname{Ind}\left(\widehat{A}_{\alpha}(t)\right)=\operatorname{Ind}\left(\widehat{A}_{\beta}(t)\right), \\
R\left(\widehat{A}_{\alpha}(t)\right)=R\left(\widehat{A}_{\beta}(t)\right) .
\end{gathered}
$$

Theorem 11. If the homogeneous equation $A(t) x_{n+1}=B(t) x_{n}$ is tractable, then the general solution is given by

$$
x_{n}=\left\{\begin{array}{ll}
\widehat{A}(t) \widehat{A}^{d}(t) q & \text { if } n=0 \\
\left(\widehat{A}^{d}(t) \widehat{B}(t)\right)^{n} q & \text { if } n=1,2, \ldots
\end{array} \quad q \in \mathbb{C}^{n}(t),\right.
$$

where $\widehat{A}(t)=(\lambda A(t)-B(t))^{-1} A(t)$ and $\widehat{B}(t)=(\lambda A(t)-$ $B(t))^{-1} B(t)$ and $\lambda \in \mathbb{C}$ is such that $(\lambda A(t)-B(t))^{-1}$ exists. 
Furthermore, $c \in \mathbb{C}^{n}$ is a consistent initial vector for if and only if $c \in R\left(\widehat{A}^{k}(t)\right)=R\left(\widehat{A}_{d}(t) A(t)\right)$, where $k=\operatorname{Ind}(\widehat{A}(t))$. In this case this unique solution subject to $x_{0}=c$ is given by $x_{n}=\left(\widehat{A}_{d}(t) \widehat{B}(t)\right)^{n} c, n=0,1,2,3, \ldots$.

Proof. Since (3) is tractable, multiplying by $(\lambda A(t)-B(t))^{-1}$ gives the equivalent equation $\widehat{A}(t) x_{n+1}=\widehat{B}(t) x_{n}$. After a similarity we get, as in the proof of Theorem 9 , that

$$
\begin{aligned}
& \left(\begin{array}{cc}
C(t) & 0 \\
0 & N(t)
\end{array}\right)\left(\begin{array}{l}
y_{n+1}^{(1)}(t) \\
y_{n+1}^{(2)}(t)
\end{array}\right) \\
& \quad=\left(\begin{array}{cc}
I+\lambda C(t) & 0 \\
0 & I+\lambda N(t)
\end{array}\right)\left(\begin{array}{l}
y_{n}^{(1)}(t) \\
y_{n}^{(2)}(t)
\end{array}\right) \\
& =\left(\begin{array}{cc}
\widehat{B}_{1} & 0 \\
0 & \widehat{B}_{2}
\end{array}\right)\left(\begin{array}{l}
y_{n}^{(1)}(t) \\
y_{n}^{(2)}(t)
\end{array}\right) .
\end{aligned}
$$

The difference equation is equivalent to the pair of equations

$$
\begin{aligned}
& C(t) y_{n+1}^{(1)}(t)=\widehat{B}_{1}(t) y_{n}^{(1)}(t), \\
& N(t) y_{n+1}^{(2)}(t)=\widehat{B}_{2}(t) y_{n}^{(2)}(t) .
\end{aligned}
$$

Since $\widehat{B}_{2}$ is invertible, the unique solution of the second equation of (25) was $y_{n+1}^{(2)}(t)=\widehat{B}_{2}^{-k}(t) N^{K} y_{n+k}^{(2)}=0$. But the first equation of (25) is consistent for any $y_{0}(t)$ and the unique solution is $y_{n}^{(1)}(t)=C^{-n}(t)\left(\widehat{B}_{1}(t)\right) y_{0}^{(1)}(t)$. In terms of the original variables we have

$$
\begin{aligned}
x_{n}= & T(t) y_{n}(t)=T(t) \\
& \cdot\left(\begin{array}{cc}
C^{-n}(t)(\widehat{B} 1(t))^{n} & 0 \\
0 & 0
\end{array}\right) T^{-1}(t) T(t) \\
& \cdot\left(\begin{array}{ll}
I & 0 \\
0 & 0
\end{array}\right) T^{-1}(t) T(t)\left(\begin{array}{l}
y_{0}^{(1)}(t) \\
y_{0}^{(2)}(t)
\end{array}\right)=\left(\widehat{A}_{d} \widehat{B}\right)^{n} q,
\end{aligned}
$$

where $q=\left(y_{0}^{(1)}(t), y_{0}^{(2)}(t)\right)^{T}$ is arbitrary.

\section{Linear Coefficient Differential Algebraic Equations}

In order to establish a sufficient and necessary condition for linear coefficient DAEs, the following lemma is important.

Lemma 12 (see [13]). Let $A, B \in \mathbb{C}^{n \times n}$. Assume that there exists $\lambda \in \mathbb{C}$, such that $(\lambda A+B)^{-1}$ exists, and let

$$
\begin{aligned}
\widehat{A}_{\lambda} & =(\lambda A+B)^{-1} A, \\
\widehat{B}_{\lambda} & =(\lambda A+B)^{-1} B .
\end{aligned}
$$

Then $\widehat{A}_{\lambda} \widehat{B}_{\lambda}=\widehat{B}_{\lambda} \widehat{A}_{\lambda}$.
Theorem 13. For $A, B \in \mathbb{C}^{n \times n}$, the homogeneous difference equation

$$
A x_{n+1}=B x_{n}
$$

is tractable if and only if there exists $\lambda \in \mathbb{C}$ such that $(\lambda A+B)^{-1}$ exists.

Proof. The proof follows from Theorem 9.

The next lemma will be used to show that the solution of the difference equation is independent of the scalar $\lambda$ which is used in the expressions $(\lambda A+B)^{-1}$ and $(\lambda A-B)^{-1}$.

Lemma 14. Assume that $A, B \in \mathbb{C}^{n \times n}$ are such that there exists $\lambda \in \mathbb{C}$ such that $(\lambda A \pm B)^{-1}$ exists. Let $\widehat{A}_{\lambda}=(\lambda A \pm B)^{-1} A$, $\widehat{B}_{\lambda}=(\lambda A \pm B)^{-1} B$. For all $\alpha, \beta \in \mathbb{C}$ for which $(\gamma A \pm B)^{-1}$ and $(\delta A \pm B)^{-1}$ exist, the following statements are true:

$$
\begin{aligned}
& \widehat{A}_{\gamma}^{d} \widehat{A}_{\gamma}=\widehat{A}_{\delta}^{d} \widehat{A}_{\delta}, \\
& \widehat{A}_{\gamma}^{d} \widehat{B}_{\gamma}=\widehat{A}_{\delta}^{d} \widehat{B}_{\delta}, \\
& \widehat{A}_{\gamma} \widehat{B}_{\gamma}^{d}=\widehat{A}_{\delta} \widehat{A}_{\delta}^{d} .
\end{aligned}
$$

At last, we give the general solution of the singular difference equation.

Theorem 15 (see [14]). If the homogeneous equation $A x_{n+1}=$ $B x_{n}$ is tractable, then the general solution is given by

$$
x_{n}=\left\{\begin{array}{ll}
\widehat{A} \widehat{A}^{d} q & \text { if } n=0 \\
\left(\widehat{A}^{d} \widehat{B}\right)^{n} q & \text { if } n=1,2, \ldots
\end{array} \quad q \in \mathbb{C}^{n}\right.
$$

where $\widehat{A}=(\lambda A-B)^{-1} A$ and $\widehat{B}=(\lambda A-B)^{-1} B$ and $\lambda \in \mathbb{C}$ is such that $(\lambda A-B)^{-1}$ exists. Furthermore, $c \in \mathbb{C}^{n}$ is a consistent initial vector if and only if $c \in R\left(\widehat{A}^{k}\right)=R\left(\widehat{A}^{d}(t) A\right)$, where $k=\operatorname{Ind}(\widehat{A})$. In this case this unique solution subject to $x_{0}=c$ is given by $x_{n}=\left(\widehat{A}^{d} \widehat{B}\right)^{n} c, n=0,1,2,3, \ldots$.

Proof. The proof follows from Theorem 11

4.1. Radau IIA Methods. We will introduce the IRK which would be applied concisely. IRK methods play an important role for the numerical solution of DAEs. Due to their onestep nature, IRK methods are potentially more efficient for these problems than multistep methods because multistep methods must be restarted, usually at low order, after every discontinuity, whereas IRK methods can restart at a higher order.

The s-stage implicit Runge-Kutta method applied to the general nonlinear DAE of the form (2) is defined by

$$
\begin{aligned}
& 0=F\left(t_{i}, X_{i}, K_{i}\right) \\
& t_{i}=t_{n-1}+c_{i} h, \quad i=1,2, \ldots, s,
\end{aligned}
$$




$$
\begin{aligned}
& X_{i}=x_{n-1}+h \sum_{j=1}^{s} a_{i j} K_{j}, \\
& x_{n}=x_{n-1}+h \sum_{i=1}^{s} b_{i} K_{i} .
\end{aligned}
$$

The method is often denoted by the shorthand notation or Butcher diagram:

$$
\begin{array}{c|c|cccc}
c & c_{1} & a_{11} & a_{11} & \cdots & a_{1 s} \\
& c_{2} & a_{21} & a_{22} & \cdots & a_{2 s} \\
\widehat{b}^{T} & \vdots & \vdots & \vdots & \ddots & \vdots \\
& c_{s} & a_{s 1} & a_{s 2} & \cdots & a_{s s} \\
\hline & b_{1} & b_{2} & \cdots & b_{s}
\end{array}
$$

4.2. Padé Approximation [15]. A differential algebraic equation has the form

$$
F(t, x, \dot{x})=0
$$

with initial values

$$
\begin{aligned}
& x\left(t_{0}\right)=x_{0}, \\
& \dot{x}\left(t_{0}\right)=x_{1},
\end{aligned}
$$

where $F$ and $x$ are vector functions for which we assumed sufficient differentiability.

We assume the solution has the form

$$
x=x_{0}+x_{1} t+e t^{2},
$$

where $e$ is a vector function which is the same size as $x_{0}$ and $x_{0}^{\prime}$. Substituting (38) into (36) and neglecting higher order term, we have the linear equation of $e$ in the form

$$
A e=B \text {, }
$$

where $A$ and $B$ are constant matrixes. Solving (39), the coefficients of $t^{2}$ can be determined. Repeating the above procedure for higher order terms, we can get the arbitrary order power series of the solutions for (2).

The power series given by above procedure can be transformed into Padé series and we have numerical solution of differential algebraic equation into (2); the specific steps can be followed by (3.1)-(3.7) in [15].

\section{Numerical Experiments}

Example 1. We consider the following differential algebraic equation:

$$
A \dot{x}+B x=0
$$

where

$$
\begin{aligned}
A & =\left(\begin{array}{ccc}
2 & 3 & 2 \\
1 & 0 & -2 \\
0 & 0 & 0
\end{array}\right), \\
B & =\left(\begin{array}{ccc}
18 & 14 & 10 \\
0 & 1 & 2 \\
-27 & -21 & -15
\end{array}\right),
\end{aligned}
$$

and we take $h=0.1$; the analytical solution of $(40)$ is $x(t)=$ $\left(1 / 6+1 / 6 e^{2 t / 3},-1 / 3+4 / 3 e^{2 t / 3}, 1 / 6-13 / 6 e^{2 t / 3}\right)^{T}$.

(1) Drazin Inverse. Suppose $t_{n+1}=t_{n}+h$; $h$ is sufficiently small. Then

$$
A \frac{x\left(t_{n+1}\right)-x\left(t_{n}\right)}{t_{n+1}-t_{n}} \approx A x\left(t_{n}\right)=-B x\left(t_{n}\right)
$$

so on the point $t_{n+1}=t_{n}+h$ the approximate value of $A x^{\prime}$ is given by

$$
A x_{n+1}=A x_{n}-h B x_{n} .
$$

Now we use the difference rule to solve the equation. Set

$$
C=A-h B=\left(\begin{array}{ccc}
0.2000 & 1.6000 & 1.0000 \\
1.0000 & -0.1000 & -2.2000 \\
2.7000 & 2.1000 & 1.5000
\end{array}\right)
$$

and then

$$
A+C=\left(\begin{array}{ccc}
2.2000 & 4.6000 & 3.0000 \\
2.0000 & -0.1000 & -4.2000 \\
2.7000 & 2.1000 & 1.5000
\end{array}\right) \text {. }
$$

$\operatorname{det}(A+C)=-33.48 \neq 0$, so $\lambda=1$ such that $(\lambda A+C)^{-1}$ exists; we can see the homogeneous equation $A x_{n+1}=C x_{n}$ is tractable. For $-A-C=-(A+C)$, we have $\lambda=-1$ such that $(\lambda A-C)^{-1}$ exists. Thus

$$
\begin{aligned}
& \widehat{A}=(-A-C) A=\left(\begin{array}{ccc}
0.5000 & 0.7769 & 0.5538 \\
-1.0000 & -1.2849 & -0.5699 \\
0.5000 & 0.4005 & -0.1989
\end{array}\right), \\
& \widehat{C}=(-A-C) C=\left(\begin{array}{ccc}
-1.5000 & -0.7769 & -0.5538 \\
1.0000 & 0.2849 & 0.5699 \\
-0.5000 & -0.4005 & -0.8011
\end{array}\right) .
\end{aligned}
$$

The eigenvalues of $\widehat{A}_{d}$ are $0,-0.5$, and -0.4839 , so that $\widehat{A}_{d}$ could be computed by Theorem 7.5.2 in [13]:

$$
\widehat{A}_{d}=\left(\begin{array}{ccc}
2.0000 & 3.1148 & 2.2296 \\
-4.0000 & -5.0815 & -2.1630 \\
2.0000 & 1.5074 & -0.9852
\end{array}\right) .
$$


According to Theorem 11, the general solution is given by

$x_{n}$

$$
=\left\{\begin{array}{l}
\left(\begin{array}{ccc}
-1.0000 & -1.5556 & -1.1111 \\
2.0000 & 2.5556 & 1.1111 \\
-1.0000 & -0.7778 & 0.4444
\end{array}\right) q \quad \text { if } n=0 \\
\left(\begin{array}{ccc}
-1.0000 & -1.5593 & -1.1185 \\
2.000 & 2.5259 & 1.0519 \\
-1.000 & -0.7296 & 0.5407
\end{array}\right) q \quad \text { if } n=1,2, \ldots,
\end{array}\right.
$$

where $q=x(0)=(1 / 3,1,-2)^{T}$, which is a consistent initial vector.

(2) Radau IIA Methods. We consider Radau IIA as follows:

\begin{tabular}{c|cc}
$\frac{1}{3}$ & $\frac{5}{12}$ & $-\frac{1}{12}$ \\
1 & $\frac{3}{4}$ & $\frac{1}{4}$ \\
\hline & $\frac{3}{4}$ & $\frac{1}{4}$
\end{tabular}

The 2-stage implicit Runge-Kutta method is applied to (40):

$$
\begin{aligned}
A K_{n, 1}+B\left(x_{n}+h\left(\frac{5}{12} K_{n, 1}-\frac{1}{12} K_{n, 2}\right)\right) & =0, \\
A K_{n, 2}+B\left(x_{n}+h\left(\frac{3}{4} K_{n, 1}-\frac{1}{4} K_{n, 2}\right)\right) & =0 .
\end{aligned}
$$

We can rewrite (50) as follows:

$$
\left(\begin{array}{cc}
A+\frac{5}{12} h B & -\frac{1}{12} h B \\
\frac{3}{4} h B & A+\frac{1}{4} h B
\end{array}\right)\left(\begin{array}{l}
K_{n, 1} \\
K_{n, 2}
\end{array}\right)=\left(\begin{array}{c}
-B x_{n} \\
-B x_{n}
\end{array}\right)
$$

where the coefficient matrix is nonsingular. So, we can compute the numerical solutions by the following equation:

$$
x_{n+1}=x_{n}+h\left(\frac{3}{4} K_{n, 1}+\frac{1}{4} K_{n, 2}\right) \text {. }
$$

(3) Padé Approximation. From initial values $x(0)=$ $(1 / 3,1,-2)^{T}$, the solutions of $(40)$ can be supposed as

$$
\begin{aligned}
& x_{1}(t)=\frac{1}{3}+e_{1} t, \\
& x_{2}(t)=1+e_{2} t, \\
& x_{3}(t)=-2+e_{3} t .
\end{aligned}
$$

Substituting (53) into (40) and neglecting higher order terms, we have

$$
\begin{aligned}
\left(2 e_{1}+3 e_{2}+2 e_{3}\right)+O(t) & =0 \\
\left(e_{1}-2 e_{3}\right)+O(t) & =0 \\
\left(9 e_{1}+7 e_{2}+5 e_{3}\right) t & =0 .
\end{aligned}
$$

The linear equation can be given in the following:

$$
\left(\begin{array}{ccc}
2 & 3 & 2 \\
1 & 0 & -2 \\
9 & 7 & 5
\end{array}\right)\left(\begin{array}{l}
e_{1} \\
e_{2} \\
e_{3}
\end{array}\right)=\left(\begin{array}{l}
0 \\
0 \\
0
\end{array}\right)
$$

Solving this linear equation we have $e=(1 / 9,8 / 9,-13 / 9)^{T}$; then the solutions of (40) can be supposed as

$$
\begin{aligned}
& x_{1}(t)=\frac{1}{3}+\frac{1}{9 t}+e_{1} t, \\
& x_{2}(t)=1+\frac{8}{9 t}+e_{2} t, \\
& x_{3}(t)=-2-\frac{13}{9 t}+e_{3} t .
\end{aligned}
$$

In the same manner, substituting (56) into (40) and neglecting higher order terms, then we have

$$
\begin{gathered}
\left(4 e_{1}+6 e_{2}+4 e_{3}\right) t+O\left(t^{2}\right)=0 \\
\left(2 e_{1}-4 e_{3}-2\right) t+O\left(t^{2}\right)=0 \\
\left(-27 e_{1}-21 e_{2}-15 e_{3}\right) t^{2}=0
\end{gathered}
$$

Similar to (53), solving this linear equation, we have $e=$ $(1 / 27,8 / 27,-13 / 27)^{T}$; then the solutions of (40) can be supposed as

$$
\begin{aligned}
& x_{1}(t)=\frac{1}{3}+\frac{1}{9 t}+\frac{1}{27 t^{2}}+e_{1} t^{3}, \\
& x_{2}(t)=1+\frac{8}{9 t}+\frac{8}{27 t^{2}}+e_{2} t^{3}, \\
& x_{3}(t)=-2-\frac{13}{9 t}-\frac{13}{27 t^{2}}+e_{3} t^{3} .
\end{aligned}
$$

Repeating the above procedure we have

$$
\begin{aligned}
x_{1}(t)= & \frac{1}{3}+\frac{1}{9 t}+\frac{1}{27 t^{2}}+\frac{2}{243 t^{3}}+\frac{1}{729 t^{4}}+\frac{2}{10935 t^{5}} \\
& +\frac{2}{98415 t^{6}}+\frac{4}{2066715 t^{7}}+\frac{1}{6200145 t^{8}} \\
& +\frac{2}{167403915 t^{9}}+O\left(t^{10}\right),
\end{aligned}
$$




$$
\begin{aligned}
& x_{2}(t)=1+\frac{8}{9 t}+\frac{8}{27 t^{2}}+\frac{16}{243 t^{3}}+\frac{8}{729 t^{4}}+\frac{16}{10935 t^{5}} \\
& -\frac{26}{98415 t^{6}}-\frac{52}{2066715 t^{7}}-\frac{13}{6200145 t^{8}} \\
& +\frac{16}{98415 t^{6}}+\frac{32}{2066715 t^{7}}+\frac{8}{6200145 t^{8}} \\
& +\frac{16}{167403915 t^{9}}+O\left(t^{10}\right) \\
& -\frac{26}{167403915 t^{9}}+O\left(t^{10}\right) \text {. } \\
& x_{3}(t)=-2-\frac{13}{9 t}-\frac{13}{27 t^{2}}-\frac{26}{243 t^{3}}-\frac{13}{729 t^{4}}-\frac{26}{10935 t^{5}} \\
& v_{1}(t)=\left[\frac{5}{4}\right]=\frac{1 / 3+1 / 81 t+4 / 243 t^{2}+1 / 1701 t^{3}+1 / 15309 t^{4}+1 / 688905 t^{5}}{1-8 / 27 t+1 / 27 t^{2}-4 / 1701 t^{3}+1 / 15309 t^{4}}, \\
& v_{2}(t)=\left[\frac{5}{4}\right]=\frac{1+16 / 27 t+17 / 243 t^{2}+44 / 5103 t^{3}+19 / 45927 t^{4}+8 / 688905 t^{5}}{1-8 / 27 t+1 / 27 t^{2}-4 / 1701 t^{3}+1 / 15309 t^{4}}, \\
& v_{3}(t)=\left[\frac{5}{4}\right]=\frac{-2-23 / 27 t-31 / 243 t^{2}-67 / 5103 t^{3}-32 / 45927 t^{4}-13 / 688905 t^{5}}{1-8 / 27 t+1 / 27 t^{2}-4 / 1701 t^{3}+1 / 15309 t^{4}} .
\end{aligned}
$$

The power series can be transformed into the following Padé

If the power series $x_{1}(t), x_{2}(t), x_{3}(t)$ converges very fast then $x_{1}(t), x_{2}(t), x_{3}(t)$ can be eliminated in Padé series.

In Tables 1-5, exact solutions, numerical solutions, and errors are illustrated.

Example 2 (see [16]). We consider the following differential algebraic equation:

$$
A(t) x^{\prime}(t)+B(t) x(t)=0,
$$

where

$$
\begin{aligned}
A & =\left(\begin{array}{llll}
1 & 0 & 0 & 0 \\
0 & 1 & 0 & 0 \\
0 & 0 & 1 & 0 \\
0 & 0 & 0 & 0
\end{array}\right), \\
B & =\left(\begin{array}{cccc}
1 & 0 & -t & 1 \\
-1 & 1 & -t^{2} & t \\
-t^{3} & t^{2} & -1 & 0 \\
t & -1 & t & -1
\end{array}\right),
\end{aligned}
$$

and we take $h=0.01$; the analytical solution of $(40)$ is $x(t)=$ $\left(e^{-t}, t e^{-t}, e^{-t}, t e^{t}\right)^{T}$.

(1) Drazin Inverse. The classical four-order Runge-Kutta method is applied to (40); we can get

$$
A(t) x_{n+1}=A(t) x_{n}+\frac{h\left[k_{1}+2 k_{2}+2 k_{3}+k_{4}\right]}{6},
$$

where

$$
\begin{aligned}
& k_{1}=-B(t) x_{n}, \\
& k_{2}=-B(t)+\frac{h k_{1}}{2}, \\
& k_{3}=-B(t)+\frac{h k_{2}}{2}, \\
& k_{4}=-B(t)+h k_{3} .
\end{aligned}
$$

Thus

$$
A(t) x_{n+1}=\left[A(t)-\left(h+\frac{h^{2}}{2}+\frac{h^{3}}{6}+\frac{h^{4}}{24}\right) B(t)\right] x_{n} \text {. }
$$
Set

Now we use the difference rule to solve the system of (40).

$$
\begin{aligned}
C(t) & =\left[A(t)-\left(h+\frac{h^{2}}{2}+\frac{h^{3}}{6}+\frac{h^{4}}{24}\right) B(t)\right] \\
& =\left(\begin{array}{cccc}
0.8948 & 0 & 0.1052 t & -0.1052 \\
0.1052 & 0.8948 & 0.1052 t^{2} & -0.1052 t \\
0.1052 t^{3} & -0.1052 t^{2} & 1.1052 & 0 \\
-0.1052 t & 0.1052 & -0.1052 t & 0.1052
\end{array}\right) ;
\end{aligned}
$$

set $\lambda=-1$; we get $\operatorname{det}(-A(t)-C(t))=0.7925 \neq 0$, so we have $\lambda=-1$ such that $(\lambda A(t)-C(t))^{-1}$ exists. Thus 
TABLE 1: Exact solution and numerical solution of $x^{(1)}(n)$.

\begin{tabular}{lcccc}
\hline$t(h=0.1)$ & $x^{(1)}(n)$ & $x_{n}^{(1)}$ of Drazin inv & $x_{n}^{(1)}$ of Radau & $x_{n}^{(1)}$ of Padé approx \\
\hline 0.1 & 0.3448231843 & 0.3443666667 & 0.3448231345 & 0.3448231842 \\
0.2 & 0.3571051353 & 0.3563563033 & 0.3571050289 & 0.3571051354 \\
0.3 & 0.3702337930 & 0.3691464551 & 0.3702336224 & 0.3702337931 \\
0.4 & 0.3842675287 & 0.3827904314 & 0.3842672855 & 0.3842675289 \\
0.5 & 0.3992687375 & 0.3973451205 & 0.3992684126 & 0.3992687378 \\
0.6 & 0.4153041163 & 0.4128712011 & 0.4153036995 & 0.4153041166 \\
0.7 & 0.4324449597 & 0.4294333945 & 0.4324444400 & 0.4324449593 \\
0.8 & 0.4507674776 & 0.4471007347 & 0.4507668426 & 0.4507674772 \\
0.9 & 0.4703531334 & 0.4659468548 & 0.4703523699 & 0.4703531335 \\
1.0 & 0.4912890068 & 0.4860502943 & 0.4912881000 & 0.4912890064 \\
\hline
\end{tabular}

TABLE 2: Exact solution and numerical solution of $x^{(2)}(n)$.

\begin{tabular}{lcccc}
\hline$t(h=0.1)$ & $x^{(2)}(n)$ & $x_{n}^{(2)}$ of Drazin inv & $x_{n}^{(2)}$ of Radau & $x_{n}^{(2)}$ of Padé approx \\
\hline 0.1 & 1.0919188077 & 1.0887666667 & 1.0919184095 & 1.0919188072 \\
0.2 & 1.1901744157 & 1.1832248233 & 1.1901735645 & 1.1901744153 \\
0.3 & 1.2952036775 & 1.2839732084 & 1.2952023127 & 1.2952036776 \\
0.4 & 1.4074735628 & 1.3914309536 & 1.4074716175 & 1.4074735624 \\
0.5 & 1.5274832334 & 1.5060450899 & 1.5274806342 & 1.5274832337 \\
0.6 & 1.6557662635 & 1.6282924326 & 1.6557629295 & 1.6557662632 \\
0.7 & 1.7928930110 & 1.7586815666 & 1.7928888531 & 1.7928930116 \\
0.8 & 1.9394731538 & 1.8977549625 & 1.9394680743 & 1.9394731533 \\
0.9 & 2.0961584005 & 2.0460912345 & 2.0961522922 & 2.0961584002 \\
1.0 & 2.2636453881 & 2.2043075487 & 2.2636381331 & 2.2636453884 \\
\hline
\end{tabular}

TABLE 3: Exact solution and numerical solution of $x^{(3)}(n)$.

\begin{tabular}{lcccc}
\hline$t(h=0.1)$ & $x^{(3)}(n)$ & $x_{n}^{(3)}$ of Drazin inv & $x_{n}^{(3)}$ of Radau & $x_{n}^{(3)}$ of Padé approx \\
\hline 0.1 & -2.1493680625 & -2.1443333333 & -2.1493674154 & -2.1493680622 \\
0.2 & -2.3090334256 & -2.2981718600 & -2.3090320423 & -2.3090334254 \\
0.3 & -2.4797059760 & -2.4622586591 & -2.4797037581 & -2.4797059762 \\
0.4 & -2.6621445395 & -2.6372765649 & -2.6621413784 & -2.6621445398 \\
0.5 & -2.8571602544 & -2.8239538938 & -2.8571560306 & -2.8571602546 \\
0.6 & -3.0656201782 & -3.0230674884 & -3.0656147604 & -3.0656201787 \\
0.7 & -3.2884511428 & -3.2354459509 & -3.2884443862 & -3.2884511424 \\
0.8 & -3.5266438749 & -3.4619730912 & -3.5266356207 & -3.5266438745 \\
0.9 & -3.7812574008 & -3.7035916057 & -3.7812474748 & -3.7812574003 \\
1.0 & -4.0534237556 & -3.9613070007 & -4.0534119663 & -4.0534237552 \\
\hline
\end{tabular}

TABLE 4: Errors $E_{n}:=\left\|x(n)-x_{n}\right\|_{2}$.

\begin{tabular}{lccc}
\hline$t(h=0.1)$ & $E_{n}$ of Drazin inverse & $E_{n}$ of Radau & $E_{n}$ of Padé approximation \\
\hline 0.1 & $5.9575917383 e-003$ & $7.6143396932 e-007$ & $5.9160779268 e-010$ \\
0.2 & $1.2916314922 e-002$ & $1.6276920132 e-006$ & $4.5825760741 e-010$ \\
0.3 & $2.0777743067 e-002$ & $2.6097631713 e-006$ & $2.4494899455 e-010$ \\
0.4 & $2.9630445273 e-002$ & $3.7196628261 e-006$ & $5.3851633972 e-010$ \\
0.5 & $3.9572170567 e-002$ & $4.9700993038 e-006$ & $4.6904157928 e-010$ \\
0.6 & $5.0709583610 e-002$ & $6.3750949076 e-006$ & $6.5574377970 e-010$ \\
0.7 & $6.3158869695 e-002$ & $7.9504631349 e-006$ & $8.2462103179 e-010$ \\
0.8 & $7.7046497345 e-002$ & $9.7126908162 e-006$ & $7.5498350599 e-010$ \\
0.9 & $8.3510065024 e-002$ & $1.1679886007 e-005$ & $5.9160780206 e-010$ \\
1.0 & $9.1969922436 e-002$ & $1.3872451324 e-005$ & $6.4031244204 e-010$ \\
\hline
\end{tabular}


TABLE 5: Errors of Drazin inverse and Radau IIA.

\begin{tabular}{llr}
\hline$t(h=0.01)$ & $E_{n}$ of Drazin inverse & $E_{n}$ of Radau IIA \\
\hline 0.01 & $1.1228248162 e-004$ & $1.0088260628 e-003$ \\
0.02 & $6.3035622437 e-004$ & $8.6145976589 e-003$ \\
0.03 & $5.5249099531 e-004$ & $1.2036909993 e-003$ \\
0.04 & $3.9265521061 e-004$ & $1.3035988488 e-003$ \\
0.05 & $1.7558562753 e-004$ & $5.5498031923 e-003$ \\
0.06 & $1.7313059801 e-004$ & $6.7699688079 e-003$ \\
0.07 & $1.2203796056 e-004$ & $7.1281533560 e-003$ \\
0.08 & $5.0551368477 e-004$ & $9.6806736561 e-003$ \\
0.09 & $6.6468312281 e-004$ & $9.9061602809 e-003$ \\
0.1 & $6.1145689285 e-004$ & $1.3892356616 e-003$ \\
\hline
\end{tabular}

$$
\begin{aligned}
& \widehat{A}(t)=(-A(t)-C(t)) A=\left(\begin{array}{cccc}
-0.5294-0.0294 t & 0.0294 & 0 & 0 \\
0.0294-0.0294 t^{2} & -0.5294+0.0294 t & 0 & 0 \\
0.0015 t^{2}+0.0264 t^{3} & -0.0264 t^{2} & -0.475 & 0 \\
-0.0294-0.5294 t+0.0015 t^{3}+0.0264 t^{4} & 0.5294-0.0264 t^{3} & -0.475 t & 0
\end{array}\right), \\
& \widehat{C}(t)=(-A(t)-C(t)) C=\left(\begin{array}{cccc}
-0.4975+0.0025 t & -0.0025 & 0 & 0 \\
-0.0025+0.0025 t^{2} & -0.4975-0.0025 t & 0 & 0 \\
-0.0025 t^{3} & 0.0025 t^{2} & -0.5025 & 0 \\
0.0025+0.5025 t-0.0025 t^{4} & -0.5025+0.0025 t^{3} & 0.4975 t & -1
\end{array}\right)
\end{aligned}
$$

so that $\widehat{A}_{d}$ could be computed by finite algorithm (13)-(15):

$$
\widehat{A}_{d}=\left(\begin{array}{cccc}
-1.8948+0.1052 t & -0.1052 & 0 & 0 \\
-0.1052+0.1052 t^{2} & -1.8948-0.1052 t & 0 & 0 \\
-0.1052 t^{3} & 0.1052 t^{2} & -2.1052 & 0 \\
0.1052-1.8948 t-0.1052 t^{4} & 1.8948+0.1052 t^{3} & -2.1052 t & 0
\end{array}\right) .
$$

According to Theorem 15, the general solution is given by

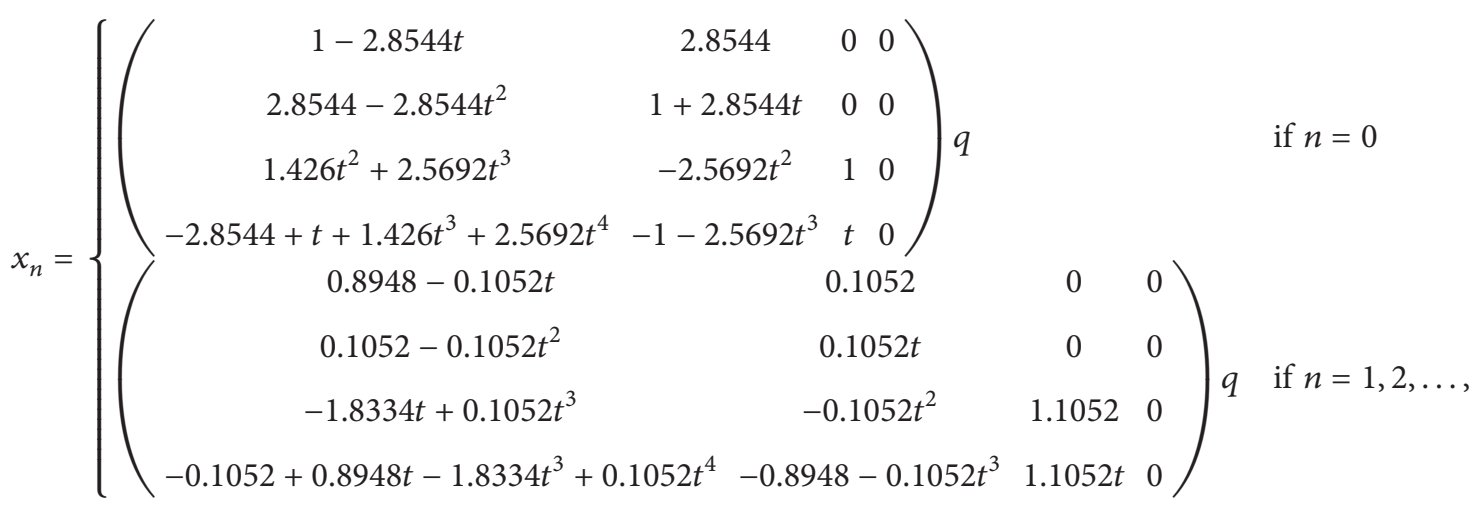

where $q \in \mathbb{C}^{n}(t)$. 
Let $q \in R(\widehat{A}(t))^{k}$; we can compute the numerical solution of (40) by (33).

(2) Radau IIA. We consider the Radau IIA as follows:

\begin{tabular}{c|cc}
$\frac{1}{3}$ & $\frac{5}{12}$ & $-\frac{1}{12}$ \\
1 & $\frac{3}{4}$ & $\frac{1}{4}$ \\
\hline & $\frac{3}{4}$ & $\frac{1}{4}$
\end{tabular}

Radau IIA is applied to (40):

$$
\begin{aligned}
A K_{n, 1}+B\left(x_{n}+h\left(\frac{5}{12} K_{n, 1}-\frac{1}{12} K_{n, 2}\right)\right) & =0, \\
A K_{n, 2}+B\left(x_{n}+h\left(\frac{3}{4} K_{n, 1}-\frac{1}{4} K_{n, 2}\right)\right) & =0 .
\end{aligned}
$$

We can rewrite (50) as follows:

$$
\left(\begin{array}{cc}
A+\frac{5}{12} h B & -\frac{1}{12} h B \\
\frac{3}{4} h B & A+\frac{1}{4} h B
\end{array}\right)\left(\begin{array}{l}
K_{n, 1} \\
K_{n, 2}
\end{array}\right)=\left(\begin{array}{c}
-B x_{n} \\
-B x_{n}
\end{array}\right)
$$

where the coefficient matrix is nonsingular. So, we can compute the numerical solutions by the following equation:

$$
x_{n+1}=x_{n}+h\left(\frac{3}{4} K_{n, 1}+\frac{1}{4} K_{n, 2}\right) \text {. }
$$

See Table 5 for errors of the numerical results. Error $E_{n}$ is defined by $E_{n}:=\left\|x(n)-x_{n}\right\|_{2}$.

\section{Conclusion}

The fundamental goal of this study has been to construct approximations to numerical solutions of linear DAEs. From Table 4, we know that the numerical solutions of Padé approximation approximate the exact solutions better than other methods, and the process of Padé approximation is easily implemented. Moreover, Drazin inverse is applied to solve the time varying differential algebraic equations. According to the obtained solutions we infer that Drazin inverse is a powerful tool for solving this kind of problems. From Table 5 we know that the precision of the Drazin inverse method is higher than the Radau IIA method, but the Drazin inverse method is implemented in more complex way than the Radau IIA method.

\section{Conflicts of Interest}

The authors declare that they have no conflicts of interest.

\section{Acknowledgments}

This work was supported by Science and Technology Commission of Shanghai Municipality (STCSM) (no. $13 \mathrm{dz2} 260400)$.

\section{References}

[1] K. E. Brenan, S. L. Campbell, and L. R. Petzold, Numerical Solution of Initial-Value Problems in Differential-Algebraic Equations, North-Holland, New York, NY, USA, 1989.

[2] S. L. Campbell, Singular System of Differential Equations, Pitman Publishing Ltd, San Francisco, 1980.

[3] S. L. Campbell, Singular System of Differential Equations II, Pitman Publishing Ltd, San Francisco, 1982.

[4] G. H. Golub and C. F. Van, Matrix Computations, Johns Hopkins University Press, Baltimore, 1983.

[5] B. Kagstrom and A. Ruhe, Matrix Pencils, Springer-Verlag, 1983.

[6] J. H. Wilkinson, "The practical significance of the drazin inverse," in Recent Applications of Generalized Inverses, S. L. Campbell, Ed., pp. 82-99, Pitman, 1982.

[7] S. L. Campbell, "One canonical form for higher-index linear time-varying singular systems," Circuits, Systems, and Signal Processing, vol. 2, no. 3, pp. 311-326, 1983.

[8] R. März, "Index-2 differential-algebraic equations," Results in Mathematics, vol. 15, pp. 149-171, 1989.

[9] R. März, "Some new results concerning index-3 differentialalgebraic equations," Journal of Mathematical Analysis and Applications, vol. 140, no. 1, pp. 177-199, 1989.

[10] R. März, "Higher-index differential-algebraic equations: analysis and numerical treatment," Numerical Analysis, vol. 24, pp. 199-222, 1990.

[11] Y. Z. Sun, "Solvability of higher index time-varying linear differential-algebraic equations," Acta Mathematica Scientia, vol. B, no. 1, pp. 77-92, 2001.

[12] J. Gao and G. Wang, "Two algorithms of Drazin inverse of a polynomial matrix," Joural of Shanghai Normal University, vol. 6, (Science Edition), 2002.

[13] S. L. Campbell and C. D. Meyer, Generalized Inverse of Linear Transformations, Pitman, Great Britain, 1979.

[14] S. L. Campbell and C. D. Meyer, Generalized Inverses of Linear Transformations, Pitman Publishing Ltd, London, 2009.

[15] C. Ercan and B. Mustafa, "Arbitrary order numerical method for solving differential-algebraic equation by Pade series," Applied Mathematics and Computation, vol. 137, pp. 57-65, 2003.

[16] S. Karimi Vanani and A. Aminataei, "Numerical solution of differential algebraic equations using a multiquadric approximation scheme," Mathematical and Computer Modelling, vol. 53, no. 5-6, pp. 659-666, 2011. 


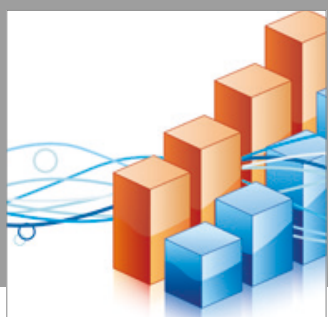

Advances in

Operations Research

vatersals

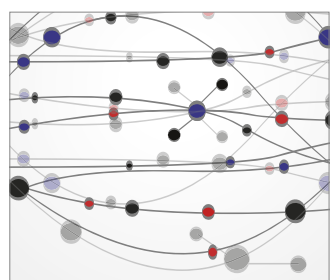

\section{The Scientific} World Journal
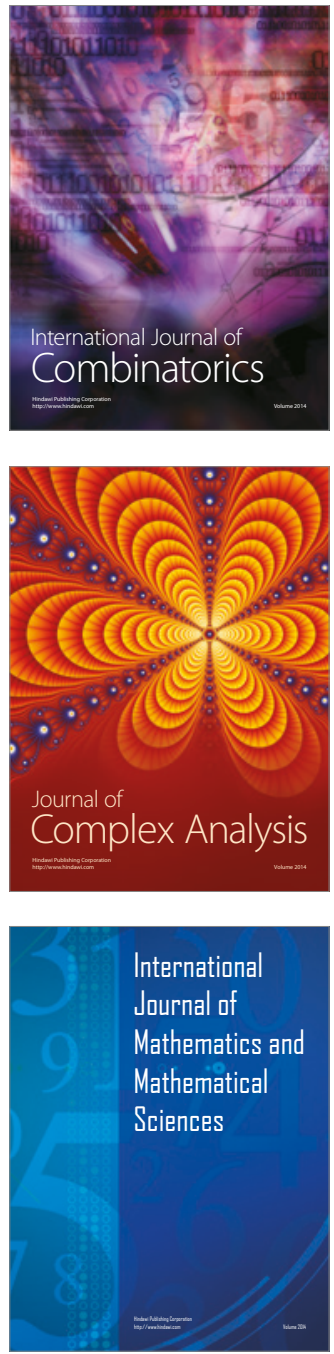
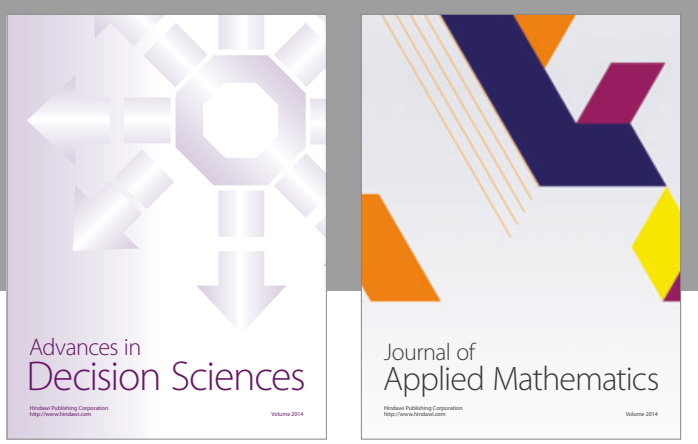

Algebra

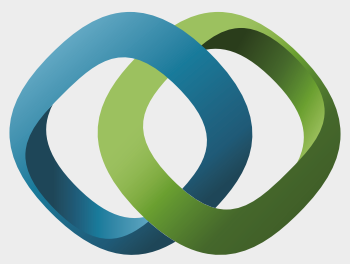

\section{Hindawi}

Submit your manuscripts at

https://www.hindawi.com
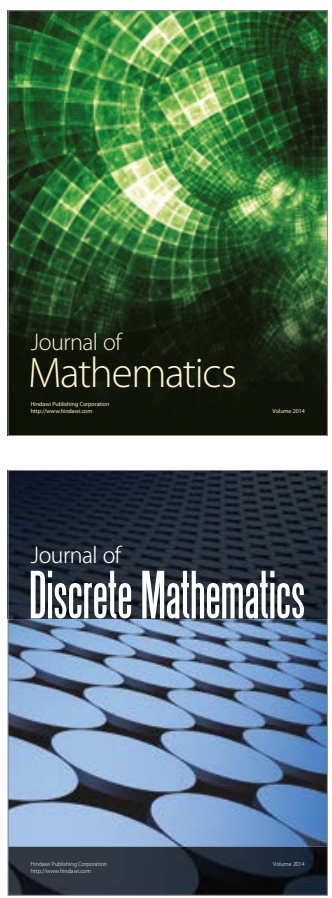

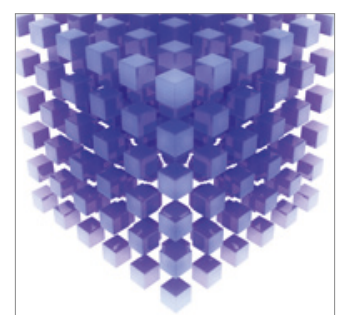

Mathematical Problems in Engineering
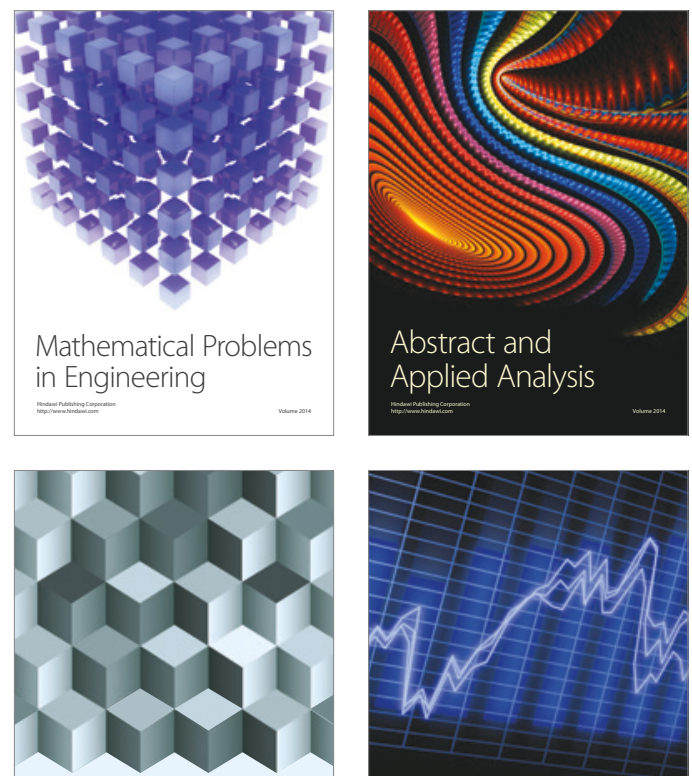

Journal of

Function Spaces

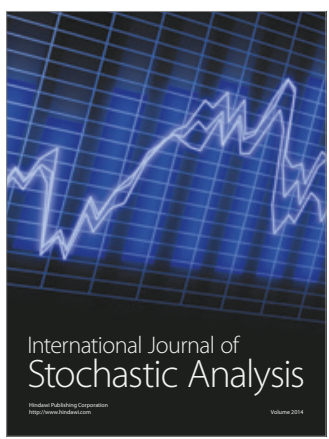

Probability and Statistics
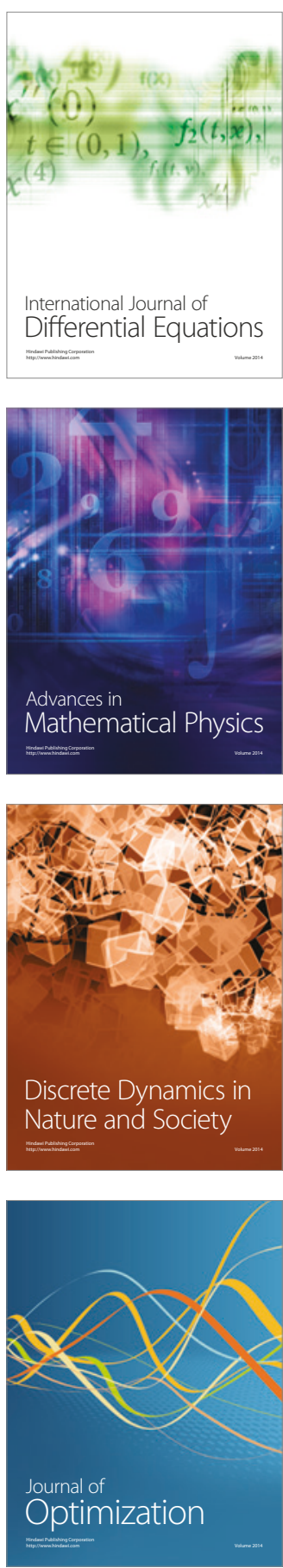